\title{
Addition and multiplication of beta-expansions in generalized Tribonacci base
}

\author{
Petr Ambrož and Zuzana Masáková and Edita Pelantová \\ Doppler Institute $\&$ Department of Mathematics \\ FNSPE, Czech Technical University, Trojanova 13, 12000 Praha 2, Czech Republic
}

received 6 Jan 2005, revised 23 Apr 2005, accepted 23 May 2005.

We study properties of $\beta$-numeration systems, where $\beta>1$ is the real root of the polynomial $x^{3}-m x^{2}-$ $x-1, m \in \mathbb{N}, m \geq 1$. We consider arithmetic operations on the set of $\beta$-integers, i.e., on the set of numbers whose greedy expansion in base $\beta$ has no fractional part. We show that the number of fractional digits arising under addition of $\beta$-integers is at most 5 for $m \geq 3$ and 6 for $m=2$, whereas under multiplication it is at most 6 for all $m \geq 2$. We thus generalize the results known for Tribonacci numeration system, i.e., for $m=1$. We summarize the combinatorial properties of infinite words naturally defined by $\beta$-integers. We point out the differences between the structure of $\beta$-integers in cases $m=1$ and $m \geq 2$.

Keywords: beta-expansion, numeration system, Pisot number

\section{Introduction}

In this paper we study the properties of a non-standard numeration system related to an irrational base $\beta$ for a class of cubic numbers $\beta$.

The definition of a numeration system based on a real number $\beta>1$ is given by the fact that every non-negative $x$ has a unique expression in the form of its $\beta$-expansion,

$$
x=\sum_{i=-\infty}^{k} a_{i} \beta^{i},
$$

where the coefficients $a_{i}$ are non-negative integers and

$$
0 \leq x-\sum_{i=n}^{k} a_{i} \beta^{i}<\beta^{n} \quad \text { for all } n \in \mathbb{Z}, n \leq k .
$$

A real number $x$ for which the $\beta$-expansion of $|x|$ is of the form $|x|=\sum_{i=0}^{k} a_{i} \beta^{i}$ is called a $\beta$-integer. The set of $\beta$-integers is denoted by $\mathbb{Z}_{\beta}$. We say that a real $x$ has a finite $\beta$-expansion, if $x$ is an element of the set

$$
\operatorname{Fin}(\beta):=\bigcup_{n=0}^{\infty} \frac{1}{\beta^{n}} \mathbb{Z}_{\beta} .
$$

1365-8050 (C) 2007 Discrete Mathematics and Theoretical Computer Science (DMTCS), Nancy, France 
If $\beta=10$, then the $\beta$-expansion of $x$ is the ordinary decimal expansion of $x$ and $\mathbb{Z}_{\beta}=\mathbb{Z}$. An equality $\mathbb{Z}_{\beta}=\mathbb{Z}$ holds for every rational integer $\beta \in \mathbb{Z}, \beta>1$.

If $\beta \notin \mathbb{Z}$, the condition (2) implies that the coefficients $a_{i}$ of the $\beta$-expansion satisfy $a_{i} \in$ $\{0,1, \ldots,\lfloor\beta\rfloor\}$ for all $i \leq k$. However, not all power series with such coefficients corresponds to a $\beta$-expansion of some real $x$. For the characterization of admissible sequences of coefficients one needs to introduce the so-called Rényi expansion of 1 ,

$$
d_{\beta}(1)=t_{1} t_{2} t_{3} \cdots, \quad \text { where } t_{1}=\lfloor\beta\rfloor \text { and } \sum_{n=2}^{\infty} \frac{t_{n}}{\beta^{n}} \text { is the } \beta \text {-expansion of } 1-\frac{t_{1}}{\beta} .
$$

A number $\beta$, for which $d_{\beta}(1)$ is eventually periodic is called a Parry number. If moreover $d_{\beta}(1)=t_{1} t_{2} t_{3} \cdots$ has only a finite number of non-zero entries $t_{i}$, then $\beta$ is called a simple Parry number and the vanishing coefficients at the end of $d_{\beta}(1)$ are omitted. Expansions of $\beta$-integers are characterized by the Parry condition [Par60]:

$\sum_{i=0}^{k} a_{i} \beta^{i}$ with $a_{i} \in\{0,1,2, \ldots\}$ is a $\beta$-expansion of some $x>0$ if and only if $a_{i} a_{i-1} \cdots a_{0} \prec$ $t_{1} t_{2} t_{3} \cdots$, i.e., $a_{i} a_{i-1} \cdots a_{0}$ is lexicographically strictly smaller than $t_{1} t_{2} t_{3} \cdots$ for any $i=k, k-$ $1, \ldots, 0$.

Unlike numeration systems with an integer base, the set $\mathbb{Z}_{\beta}$ of $\beta$-integers is not closed under addition and multiplication if $\beta \notin \mathbb{Z}$. However, this does not exclude that $\operatorname{Fin}(\beta)$ is a ring. A necessary condition so that $\operatorname{Fin}(\beta)$ is closed under addition and multiplication is that $\beta$ is a Pisot-Vijayaraghavan (PV) number and a simple Parry number [Aki00, FS92]. Recall that PV numbers are defined as real algebraic integers greater than 1 such that their conjugates are inside the unit complex circle. It is known that every PV number is a Parry number.

Some sufficient conditions on a simple Parry number $\beta$ so that $\operatorname{Fin}(\beta)$ is a ring are also known ${ }^{(i)}$. In [FS92] it is shown that if $d_{\beta}(1)=t_{1} t_{2} \cdots t_{m}$ satisfies $t_{1} \geq t_{2} \geq \cdots \geq t_{m}>0$ then $\operatorname{Fin}(\beta)$ is a ring. Hollander [Hol96] has shown that $t_{1}>t_{2}+t_{3}+\cdots t_{m}$ is a sufficient condition so that $\operatorname{Fin}(\beta)$ is a ring. For cubic PV units $\beta$ Akiyama [Aki00] has shown that $\operatorname{Fin}(\beta)$ is a ring if and only if $\beta$ is a simple Parry number, i.e., $d_{\beta}(1)$ is finite. A weaker form of the condition $(\mathrm{F})$ is the so-called weak finiteness property, studied in [ARS04].

For performing the arithmetic operations in the numeration system based on $\beta$ it is important not only to know that the result has a finite fractional part, but to know the length of the fractional part. We therefore study the quantities

$$
\begin{aligned}
& L_{\oplus}=L_{\oplus}(\beta):=\min \left\{n \in \mathbb{N}_{0} \mid \forall x, y \in \mathbb{Z}_{\beta}, x+y \in \operatorname{Fin}(\beta) \Rightarrow x+y \in \beta^{-n} \mathbb{Z}_{\beta}\right\}, \\
& L_{\otimes}=L_{\otimes}(\beta):=\min \left\{n \in \mathbb{N}_{0} \mid \forall x, y \in \mathbb{Z}_{\beta}, x y \in \operatorname{Fin}(\beta) \Rightarrow x y \in \beta^{-n} \mathbb{Z}_{\beta}\right\} .
\end{aligned}
$$

In [Aki99] it is shown that for $\beta$ a PV number, one has $L_{\oplus}<+\infty$. In [GMP04] the same result is given for $L_{\otimes}$. First known values for $L_{\oplus}, L_{\otimes}$ have been provided in [BFGK98] for quadratic PV units. Article [GMP04] states exact values and estimates on $L_{\oplus}, L_{\otimes}$ for arbitrary quadratic PV numbers. The first cubic case was studied by Messaoudi. In [Mes00] he shows for the so-called Tribonacci numeration system, i.e., for the base $\beta$ with minimal polynomial $x^{3}-x^{2}-x-1$, that $L_{\otimes} \leq 9$, and states the conjecture of Arnoux that $L_{\otimes}=3$. In [Mes02] he improves the upper

(i) We say that the number $\beta$ has Property (F) if the set $\operatorname{Fin}(\beta)$ is a ring 
bound to $L_{\otimes} \leq 6$. In [AFMP03] it is shown that $4 \leq L_{\otimes} \leq 5$ and $5 \leq L_{\oplus} \leq 6$. Final exact value $L_{\oplus}=5$ has been given with an elegant proof by Bernat [Ber05].

In this paper we study a class of cubic units $\beta>1$, roots of

$$
x^{3}=m x^{2}+x+1, \quad m \in \mathbb{N} .
$$

We call such base $\beta$ the generalized Tribonacci number, since the Tribonacci case is obtained for $m=1$. The equation $x^{3}=m x^{2}+x+1$ has a unique real solution $\beta$. It satisfies $m<\beta<m+1$. The other two roots $\beta^{\prime}$ and $\beta^{\prime \prime}$ are mutually complex conjugates $\left|\beta^{\prime}\right|=\left|\beta^{\prime \prime}\right|<1$. Obviously, we have

$$
\beta \beta^{\prime} \beta^{\prime \prime}=1, \quad \beta+\beta^{\prime}+\beta^{\prime \prime}=m .
$$

The Rényi expansion of 1 is $d_{\beta}(1)=m 11$. Therefore the digits in a $\beta$-expansion take values in $\{0,1, \ldots, m\}$ and the Parry condition implies for $a_{0}, a_{1}, \ldots, a_{k} \in \mathbb{N}_{0}$ that

$$
\sum_{i=0}^{k} a_{i} \beta^{i} \text { is a } \beta \text {-expansion } \Longleftrightarrow a_{i} a_{i-1} a_{i-2} \prec m 11 \quad \text { for all } i=2,3, \ldots, k \text {. }
$$

Due to the results of Frougny and Solomyak [FS92] or Akiyama [Aki99] the set Fin $(\beta)$ is a ring for all $m$.

Our aim is to provide estimates on the quantities $L_{\oplus}(\beta), L_{\otimes}(\beta)$ for these generalized Tribonacci numbers $\beta$. Since the Tribonacci case $m=1$ has been already solved, we consider $m \geq 2$.

Lower bounds on $L_{\oplus}(\beta), L_{\otimes}(\beta)$ can be clearly obtained as the length of the fractional part of the sum, respectively product of some elements $x, y \in \mathbb{Z}_{\beta}$.

Proposition 1 Let $\beta$ be the real root of $x^{3}=m x^{2}+x+1$. Then

$$
L_{\oplus}(\beta) \geq\left\{\begin{array}{l}
5 \quad \text { for } m=2, \\
4 \quad \text { for } m \geq 3,
\end{array} \quad L_{\otimes}(\beta) \geq 4 \text { for } m \geq 2 .\right.
$$

Proof: We have for $m=2$,

$$
\begin{aligned}
& \left(\beta^{4}+\beta^{2}+2 \beta\right)-\left(\beta^{3}+2\right)=2 \beta^{3}+2+\beta^{-3}+\beta^{-4}+\beta^{-5}, \\
& 2 \times\left(\beta^{6}+2 \beta^{5}+2 \beta^{3}+\beta^{2}+2\right)=\beta^{7}+\beta^{6}+2 \beta^{4}+2 \beta+\beta^{-2}+2 \beta^{-3}+\beta^{-4},
\end{aligned}
$$

and for $m \geq 3$,

$$
\begin{aligned}
\left(m \beta^{3}+m\right)+\left(m \beta^{3}+m\right)= & 2 \times\left(m \beta^{3}+m\right)= \\
= & \beta^{4}+(m-1) \beta^{3}+(m-1) \beta^{2}+2 \beta+ \\
& \quad+(m-3) \beta^{-1}+(m-1) \beta^{-2}+2 \beta^{-3}+\beta^{-4} .
\end{aligned}
$$


The main result of this paper is providing the upper bounds on $L_{\oplus}(\beta)$ and $L_{\otimes}(\beta)$. Using a specific method of estimation, which will be explained in the next section, we obtain $5 \leq L_{\oplus} \leq 6$, for $m=2$, and $4 \leq L_{\oplus} \leq 5$, for $m \geq 3$, (Theorem 4). Let us mention that for two special $m$ the exact value of $L_{\oplus}(\beta)$ is known [Ber05], namely for $m=2$ is $L_{\oplus}(\beta)=5$ and for $m=3$ is $L_{\oplus}(\beta)=4$. Further, we study $L_{\otimes}(\beta)$. We establish $4 \leq L_{\otimes} \leq 6$ for $m \geq 2$, (Theorem 5 ). Note that the exact value of $L_{\otimes}(\beta)$ is not known even in Tribonacci case $m=1$.

The proofs of Theorems 4 and 5 use real quadratic forms and their differences. It is noteworthy that the similar computation was the bottleneck of [AS98] and [Gji01] in studying topology of Thurston-Rauzy fractals associated with $\beta$-expansions.

Although we study the numeration systems mainly from the arithmetical point of view, its combinatorial properties are also very interesting. At the end of the paper we present some combinatorial properties of the set of $\beta$-integers which can be derived from general results in the literature for the class of generalized Tribonacci numbers.

\section{Methods of upper estimation of $L_{\oplus}(\beta), L_{\otimes}(\beta)$}

Let us present the methods for finding upper estimates of $L_{\oplus}, L_{\otimes}$ and the one chosen for the class of generalized Tribonacci numbers explain in detail. Two methods for estimation of $L_{\oplus}, L_{\otimes}$ are known. First of them uses the so-called Meyer property of the set of $\beta$-integers, namely that

$$
\mathbb{Z}_{\beta}+\mathbb{Z}_{\beta} \subset \mathbb{Z}_{\beta}+F \quad \text { for a finite set } F .
$$

In [ABF04] one studies the question of minimizing the finite set $F$. For multiplication, one has a similar property [GMP04]: If $\beta$ is a PV number, then

$$
\mathbb{Z}_{\beta} \cdot \mathbb{Z}_{\beta} \subset \mathbb{Z}_{\beta}+G \quad \text { for a finite set } G .
$$

This method is used in [AFMP03] to find values of $L_{\oplus}(\beta), L_{\otimes}(\beta)$ where $\beta$ is a PV number, solution of the equation $x^{3}=25 x^{2}+15 x+2$.

The second much more used method for estimation of $L_{\oplus}, L_{\otimes}$ is based on the following theorem. Several versions of this method are employed in [AFMP03, Ber05, GMP04, Mes00, Mes02].

Theorem 2 ([GMP04]) Let $\beta>1$ be an algebraic number, and let $\beta^{\prime}$ be its conjugate. For $z \in \mathbb{Q}_{\beta}$ we denote by $z^{\prime}$ the image of $z$ under the field isomorphism $: \mathbb{Q}(\beta) \rightarrow \mathbb{Q}\left(\beta^{\prime}\right)$. If

$$
\begin{aligned}
& H:=\sup \left\{\left|z^{\prime}\right| \mid z \in \mathbb{Z}_{\beta}\right\}<+\infty, \\
& K:=\inf \left\{\left|z^{\prime}\right| \mid z \in \mathbb{Z}_{\beta} \backslash \beta \mathbb{Z}_{\beta}\right\}>0,
\end{aligned}
$$

then

$$
\left(\frac{1}{\left|\beta^{\prime}\right|}\right)^{L_{\oplus}}<\frac{2 H}{K} \quad \text { and } \quad\left(\frac{1}{\left|\beta^{\prime}\right|}\right)^{L_{\otimes}}<\frac{H^{2}}{K} .
$$

In the above theorem we require existence of at least one conjugate of $\beta$ such that the constant $H$ is finite and $K$ is positive. The former is ensured if $\left|\beta^{\prime}\right|<1$. To decide whether $K>0$ or $K=0$ is much more complicated. In the paper [AFMP03] there is given a sufficient condition for a number $\beta$ so that $K=0$. An example for such number $\beta$ is the already mentioned solution of 
the equation $x^{3}=25 x^{2}+15 x+2$. Indeed, for such $\beta$ one cannot employ Theorem 2 . A sufficient condition for $\beta$ so that $K>0$ was given by Akiyama [Aki00]. Akiyama proved that for $\beta \mathrm{PV}$ unit such that $\operatorname{Fin}(\beta)$ is a ring, the origin is an inner point of the central tile in the conjugated plane, i.e., of the closure of the set $\left\{z^{\prime} \mid z \in \mathbb{Z}_{\beta}\right\}$. This implies $K$ is positive for all conjugates of $\beta$. In such a case one chooses the conjugate which provides the best estimates on $L_{\oplus}, L_{\otimes}$.

Our aim is to provide estimates on the quantities $L_{\oplus}(\beta), L_{\otimes}(\beta)$ for the generalized Tribonacci numbers $\beta$. We use the method based on Theorem 2. Recall that one has to choose a suitable conjugate of $\beta$. However, in the case of generalized Tribonacci numbers, both conjugates of $\beta$ have the same modulus $<1$, and thus both of them will provide the same values of constants $H, K$. Moreover, since $\beta$ is a cubic PV unit with finite Rényi expansion of 1 , set $\operatorname{Fin}(\beta)$ is a ring and therefore constant $K$ in Theorem 2 is positive.

It is a difficult problem to determine the exact values of $H$ and $K$. However, in order to obtain bounds on $L_{\oplus}$ and $L_{\otimes}$ it suffices to find some "reasonable" upper estimate on $H$ and lower estimate on $K$.

Upper estimate on $\boldsymbol{H}$. For a given $p \in \mathbb{N}$, every $z \in \mathbb{Z}_{\beta}$ can be written as

$$
z=\left(a_{0}+a_{1} \beta+\cdots+a_{p-1} \beta^{p-1}\right)+\beta^{p}\left(a_{p}+\cdots+a_{2 p-1} \beta^{p-1}\right)+\ldots,
$$

with the integer coefficients satisfying the admissibility condition $a_{i} a_{i-1} a_{i-2} \prec m 11$. Using triangular inequality, the modulus of the conjugate $z^{\prime}$ can be estimated by

$$
\left|z^{\prime}\right| \leq\left(1+\left|\beta^{\prime}\right|^{p}+\left|\beta^{\prime}\right|^{2 p}+\ldots\right) \operatorname{Max}_{p}=\frac{\operatorname{Max}_{p}}{1-\left|\beta^{\prime}\right|^{p}}=: H_{p}
$$

where

We therefore have

$$
\operatorname{Max}_{p}:=\max \left\{\left|z^{\prime}\right|\left|z \in \mathbb{Z}_{\beta},\right| z \mid<\beta^{p}\right\}
$$

$$
H \leq H_{p} \quad \text { for all } p \in \mathbb{N} .
$$

Lower estimate on $\boldsymbol{K}$. For a given $p \in \mathbb{N}$, and for all $z \in \mathbb{Z}_{\beta}$ we have

$$
\left|z^{\prime}\right| \geq\left|a_{0}+a_{1} \beta+\cdots+a_{p-1} \beta^{p-1}\right|-\left|\beta^{\prime}\right|^{p}\left|a_{p}+a_{p+1} \beta^{\prime}+\ldots\right| .
$$

Note that the last term on the right hand side is a modulus of a $\beta$-expansion of a conjugate of some $x \in \mathbb{Z}_{\beta}$ and therefore it is bounded by $H$ (or $H_{q}$ for some $q \in \mathbb{N}$ ). With the notation

$$
\operatorname{Min}_{p}:=\min \left\{\left|z^{\prime}\right|\left|z \in \mathbb{Z}_{\beta} \backslash \beta \mathbb{Z}_{\beta},\right| z \mid<\beta^{p}\right\},
$$

we obtain an estimate

$$
K \geq K_{p, q}:=\operatorname{Min}_{p}-\left|\beta^{\prime}\right|^{p} H_{q} \quad \text { for all } p, q \in \mathbb{N} .
$$

We see that the problem of the upper bounds on $L_{\oplus}$ and $L_{\otimes}$ has been reduced to the problem

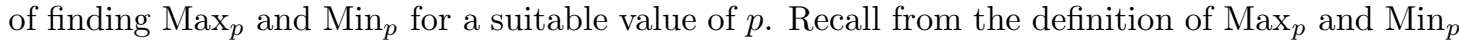
(equations (21) and (24)) that both of them are calculated using the modulus $\left|z^{\prime}\right|$ of the image 
of $\beta$-integers $|z|<\beta^{p}$ under the field morphism. It turns more convenient to study the square of this quantity.

Let us consider a $\beta$-integer $z$, such that $|z|<\beta^{p}$, i.e., $z$ is of the form $z=a_{0}+a_{1} \beta+a_{2} \beta^{2}+\cdots+$ $a_{p-1} \beta^{p-1}$, where the integer coefficients $a_{i}$ satisfy the admissibility condition $a_{i} a_{i-1} a_{i-2} \prec m 11$. Since the conjugates of $\beta$ are mutually complex conjugated we have

$$
\left|z^{\prime}\right|^{2}=z^{\prime} z^{\prime \prime}=\sum_{i=0}^{p-1} a_{i}{\beta^{\prime}}^{i} \sum_{j=0}^{p-1} a_{j} \beta^{\prime \prime j}=\sum_{i=0}^{p-1} \sum_{j=0}^{p-1} a_{i} a_{j}{\beta^{\prime}}^{i}{\beta^{\prime \prime}}^{j} .
$$

We use the relation $\beta \beta^{\prime} \beta^{\prime \prime}=1$ to derive for $j>i$,

$$
a_{i} a_{j}{\beta^{\prime}}^{i}{\beta^{\prime \prime j}}^{j}+a_{j} a_{i}{\beta^{\prime j}}^{j}{\beta^{\prime \prime}}^{i}=a_{i} a_{j} \beta^{\prime j-i} \frac{1}{\beta^{i}}+a_{i} a_{j} \beta^{\prime \prime j-i} \frac{1}{\beta^{i}}=\frac{a_{i} a_{j}}{\beta^{i}} 2 \Re\left(\beta^{\prime j-i}\right) .
$$

Substituting this to the expression for $\left|z^{\prime}\right|^{2}$, we obtain

$$
\left|z^{\prime}\right|^{2}=\sum_{k=0}^{p-1} \frac{a_{k}^{2}}{\beta^{k}}+\sum_{0 \leq i<j \leq p-1} \frac{a_{i} a_{j}}{\beta^{i}} 2 \Re\left(\beta^{j-i}\right) .
$$

Therefore, $\left|z^{\prime}\right|^{2}$ is a real quadratic form of integer variables $a_{0}, a_{1}, \ldots, a_{p-1}$. In order to express it in a simpler form, we need to determine the coefficients $2 \Re\left(\beta^{\prime j-i}\right)$. Let us denote $c_{k}:=2 \Re\left({\beta^{\prime}}^{k}\right)$. For calculation of $c_{k}$ we find a recurrent formula,

$$
\begin{aligned}
c_{k} c_{1}=\left(\beta^{\prime k}+\beta^{\prime \prime k}\right)\left(\beta^{\prime}+\beta^{\prime \prime}\right) & =\beta^{\prime k+1}+\beta^{\prime k} \beta^{\prime \prime}+\beta^{\prime \prime k} \beta^{\prime}+\beta^{\prime \prime k+1} \\
& =c_{k+1}+\beta^{\prime} \beta^{\prime \prime}\left(\beta^{\prime k-1}+\beta^{\prime \prime k-1}\right) \\
& =c_{k+1}+\frac{1}{\beta} c_{k-1}
\end{aligned}
$$

Let us enumerate several initial coefficients,

$$
\begin{aligned}
& c_{0}=2, \\
& c_{1}=-\left(\frac{1}{\beta}+\frac{1}{\beta^{2}}\right), \\
& c_{2}=-\left(\frac{2}{\beta}-\frac{1}{\beta^{2}}-\frac{2}{\beta^{3}}-\frac{1}{\beta^{4}}\right), \\
& c_{3}=\frac{3}{\beta^{2}}+\frac{2}{\beta^{3}}-\frac{3}{\beta^{4}}-\frac{3}{\beta^{5}}-\frac{1}{\beta^{6}}, \\
& c_{4}=\frac{2}{\beta^{2}}-\frac{4}{\beta^{3}}-\frac{7}{\beta^{4}}+\frac{6}{\beta^{6}}+\frac{4}{\beta^{7}}+\frac{1}{\beta^{8}} .
\end{aligned}
$$

Note that for $m \geq 3$ the coefficients satisfy $c_{1}<0, c_{2}<0, c_{3}>0$ and $c_{4}>0$.

Our aim is to find $\operatorname{Max}_{p}$ and $\operatorname{Min}_{q}$ for suitable $p, q$ so that the bounds on $L_{\oplus}, L_{\otimes}$ obtained by Theorem 2 are best possible. Computer experiments show that optimal constants are $H_{3}, K_{5,3}$, 
that is to say the values of $H_{n}$ and $K_{m, n}$ change too little with increasing $n, m$ and do not provide better estimates on the values of $L_{\oplus}$ and $L_{\otimes}$. Thus we need to calculate $\mathrm{Max}_{3}$ and $\mathrm{Min}_{5}$.

Since we are interested in $\operatorname{Max}_{3}$, the real quadratic form $\left|z^{\prime}\right|^{2}$ to be examined simplifies to

$$
Q_{1}\left(a_{2}, a_{1}, a_{0}\right):=\left|z^{\prime}\right|^{2}=a_{0}^{2}+\frac{a_{1}^{2}}{\beta}+\frac{a_{2}^{2}}{\beta^{2}}+a_{0} a_{1} c_{1}+a_{0} a_{2} c_{2}+\frac{a_{1} a_{2}}{\beta} c_{1} .
$$

In order to calculate the value of $\mathrm{Max}_{3}$ we have to find the maximum of the quadratic form $Q_{1}\left(a_{2}, a_{1}, a_{0}\right)$ on the set

$$
\mathcal{S}_{1}:=\left\{\left(a_{2}, a_{1}, a_{0}\right) \in \mathbb{Z}^{3} \mid 0 \leq a_{i} \leq m, a_{2} a_{1} a_{0} \prec m 11\right\}
$$

Similarly, for calculation of $\mathrm{Min}_{5}$, we use the quadratic form

$$
\begin{aligned}
& Q_{2}\left(a_{4}, a_{3}, a_{2}, a_{1}, a_{0}\right):=\left|z^{\prime}\right|^{2}=a_{0}^{2}+\frac{a_{1}^{2}}{\beta}+\frac{a_{2}^{2}}{\beta^{2}}+\frac{a_{3}^{2}}{\beta^{3}}+\frac{a_{4}^{2}}{\beta^{4}}+a_{0} a_{1} c_{1}+a_{0} a_{2} c_{2}+ \\
&+a_{0} a_{3} c_{3}+a_{0} a_{4} c_{4}+\frac{a_{1} a_{2}}{\beta} c_{1}+\frac{a_{1} a_{3}}{\beta} c_{2}+\frac{a_{1} a_{4}}{\beta} c_{3}+\frac{a_{2} a_{3}}{\beta^{2}} c_{1}+\frac{a_{2} a_{4}}{\beta^{2}} c_{2}+\frac{a_{3} a_{4}}{\beta^{3}} c_{1} .
\end{aligned}
$$

We have to find a minimum of this quadratic form on the set

$$
\mathcal{S}:=\left\{\left(a_{4}, a_{3}, a_{2}, a_{1}, a_{0}\right) \in \mathbb{Z}^{5} \mid m \geq a_{i} \geq 0, a_{0}>0 \text { and } a_{k+2} a_{k+1} a_{k} \prec m 11\right\} .
$$

The condition $a_{0} \neq 0$ in the definition of set $\mathcal{S}$ corresponds to the condition $z \in \mathbb{Z}_{\beta} \backslash \beta \mathbb{Z}_{\beta}$ in the definition of $\operatorname{Min}_{p}$.

For finding the extremal values of the quadratic forms, we inspect the first differences of the quadratic form for each variable $a_{i}$, each time fixing all yet appointed values of the variables. The difference in a variable $a_{i}$ will be denoted

$$
\Delta_{a_{i}}:=Q\left(\ldots, a_{i}+1, \ldots\right)-Q\left(\ldots, a_{i}, \ldots\right)
$$

where $a_{i} \leq m-1$ and the variables $a_{j}, j \neq i$ take values at most $m$. Using this method we further prove the following result.

Proposition 3 Let $\beta>1$ be the real root of the equation $x^{3}=m x^{2}+x+1$ for $m \in \mathbb{N}, m \geq 2$. Then

$$
\begin{aligned}
& \operatorname{Max}_{3}=\max \left\{\left|z^{\prime}\right|\left|z \in \mathbb{Z}_{\beta},\right| z \mid<\beta^{3}\right\}=m, \\
& \operatorname{Min}_{5}=\min \left\{\left|z^{\prime}\right|\left|z \in \mathbb{Z}_{\beta} \backslash \beta \mathbb{Z}_{\beta},\right| z \mid<\beta^{5}\right\}= \begin{cases}\beta^{-2}(\beta+1) & \text { for } m \geq 3 \\
\left|1+2 \beta^{\prime 2}+2{\beta^{\prime}}^{4}\right| & \text { for } m=2 .\end{cases}
\end{aligned}
$$

Since the proof of this proposition mainly consists of a rather technical analysis of the behavior of the quadratic forms $Q_{1}, Q_{2}$, we postpone it to a separate Section 4 . 


\section{The main result}

In the preceding sections we have found the values of $\operatorname{Max}_{3}$ and $\operatorname{Min}_{5}$. According to the definitions (20) and (25) we have for $m \geq 3$,

$$
H_{3}=\frac{\operatorname{Max}_{3}}{1-\left|\beta^{\prime}\right|^{3}}=\frac{m \beta^{3 / 2}}{\beta^{3 / 2}-1}
$$

and

$$
K_{5,3}=\operatorname{Min}_{5}-\left|\beta^{\prime}\right|^{5} H_{3}=\frac{\beta+1}{\beta^{2}}-\frac{m}{\beta\left(\beta^{3 / 2}-1\right)} .
$$

Now, we will use these values to obtain upper estimates on the value of $L_{\oplus}$.

Theorem 4 Let $\beta>1$ be the real root of the equation $x^{3}=m x^{2}+x+1$ for $m \in \mathbb{N}$. Then

$$
\begin{array}{ll}
4 \leq L_{\oplus}(\beta) \leq 5 & \text { for } m \geq 3, \\
5 \leq L_{\oplus}(\beta) \leq 6 & \text { for } m=2 .
\end{array}
$$

Proof: The upper bound $L_{\oplus} \leq 6$ for $m=2$ can be easily checked numerically. Since

$$
\frac{2 H}{K}<\frac{2 H_{3}}{K_{5,3}} \simeq 18.4596<\left(\frac{1}{\left|\beta^{\prime}\right|}\right)^{7} \simeq 26.3628,
$$

according to the Theorem 2 we have $L_{\oplus} \leq 6$.

Let us assume $m \geq 3$. Again according to the Theorem 2 we have

$$
\left(\frac{1}{\left|\beta^{\prime}\right|}\right)^{L_{\oplus}}<\frac{2 H}{K} \leq \frac{2 H_{3}}{K_{5,3}} .
$$

We will prove the inequality

$$
\frac{2 H_{3}}{K_{5,3}}=\frac{2 m \beta^{3 / 2}}{\beta^{3 / 2}-1} \frac{1}{\frac{\beta+1}{\beta^{2}}-\frac{m}{\beta\left(\beta^{3 / 2}-1\right)}}<\beta^{3}=\frac{1}{\left|\beta^{\prime}\right|^{6}},
$$

which implies $L_{\oplus} \leq 5$.

After a few simple operations the inequality (44) is transformed into

$$
2 m \beta^{1 / 2}+m \beta<(\beta+1)\left(\beta^{3 / 2}-1\right) .
$$

For $m \geq 4$ the proof of the inequality (45) will be based on the fact that $\beta>\beta^{1 / 2}+2$, which holds since

$$
\beta>\beta^{1 / 2}+2 \Leftrightarrow(\beta-2)^{2}>\beta \Leftrightarrow m \beta+1+\frac{1}{\beta}-4 \beta+4>\beta .
$$

Now we estimate the left hand side of (45)

$$
\begin{aligned}
& m \beta^{1 / 2}\left(2+\beta^{1 / 2}\right)<\beta^{1 / 2} m \beta<\beta^{1 / 2}\left(m \beta+\frac{1}{\beta}\right)=\beta^{1 / 2}\left(\beta^{2}-1\right)= \\
& =\beta^{1 / 2}(\beta-1)(\beta+1)=(\beta+1)\left(\beta^{3 / 2}-\beta^{1 / 2}\right)<(\beta+1)\left(\beta^{3 / 2}-1\right),
\end{aligned}
$$


which proves (45).

In the omitted case $m=3$, the inequality (45) can be proved directly.

The following theorem yields the main result for multiplication - the estimates of the value of the coefficient $L_{\otimes}$.

Theorem 5 Let $\beta>1$ be the real root of the equation $x^{3}=m x^{2}+x+1$ for $m \in \mathbb{N}, m \geq 2$. Then

$$
4 \leq L_{\otimes}(\beta) \leq 6
$$

Proof: Let us assume $m \geq 3$. According to the Theorem 2 it suffices to show

$$
\frac{H^{2}}{K} \leq \frac{H_{3}^{2}}{K_{5,3}}=\frac{m 2}{\left(1-\beta^{-3 / 2}\right)^{2}} \frac{1}{\frac{\beta+1}{\beta^{2}}-\beta^{-5 / 2} \frac{m}{1-\beta^{-3 / 2}}}<\frac{1}{\left|\beta^{\prime}\right|^{7}}
$$

which can be transformed into

$$
(1+m) \beta^{3}+\beta^{2}+(m+2) \beta+1>\left((m+2) \beta+m^{2}+2\right) \beta^{3 / 2} .
$$

By $m \leq \beta<m+1$ we can estimate the left hand side of (50) as

$$
(1+m) \beta^{3}+\beta^{2}+(m+2) \beta+1 \geq(1+m) m^{3}+m^{2}+(m+2) m+1 \geq m^{3}(1+m)+m^{2},
$$

whereas the right hand side as

$$
\left((m+2) \beta+m^{2}+2\right) \beta^{3 / 2}<\left((m+2)(m+1)+m^{2}+2\right)(m+1)^{3 / 2} \leq 3 m^{2}(m+1)^{3 / 2},
$$

where the last inequality holds for $m \geq 4$. The omitted cases will be treated separately at the end of the proof.

Using last two estimates we have

$$
3 m^{2}(m+1)^{3 / 2} \leq m^{2}+m^{3}(1+m) .
$$

It is easy to check that this inequality holds for $m \geq 10$.

Since our estimates were too rough to check the validity of (50) for $m \leq 9$, we verified the proposition numerically for these cases as well as for the case $m=2$ which was omitted at the beginning of the proof.

Let us mention that computer experiments support the hypothesis that $L_{\otimes}(\beta)=4$ for $m \geq 2$.

\section{Proof of Proposition 3}

In this section we provide the proof of Proposition 3. For that we will use the notions of quadratic forms introduced in Section 2. We start with determining the value of $\mathrm{Min}_{5}$, since the results will be used in the other part of the proof for $\operatorname{Max}_{3}$. 
Determining $\operatorname{Min}_{5}$. In the case $m=2$ the set $\mathcal{S}_{2}$ defined in (34) has only 79 elements. It is easy to enumerate them one by one and to find the value of $\mathrm{Min}_{5}$. Therefore, from now we will consider $m \geq 3$.

1) Difference $\Delta_{a_{0}}$. The inspected first difference of the quadratic form $Q_{2}$ is

$$
\Delta_{a_{0}}=2 a_{0}+1+c_{1} a_{1}+c_{2} a_{2}+c_{3} a_{3}+c_{4} a_{4} .
$$

Since $a_{0} \geq 1, c_{1}<0, c_{2}<0, c_{3}>0, c_{4}>0$ and $a_{1}, \ldots, a_{4} \in\{0, \ldots, m\}$ we have

$$
\Delta_{a_{0}} \geq 3+m c_{1}+m c_{2}=3-m\left(\frac{1}{\beta}+\frac{1}{\beta^{2}}\right)-m\left(\frac{2}{\beta}-\frac{1}{\beta^{2}}-\frac{2}{\beta^{3}}-\frac{1}{\beta^{4}}\right) \geq 3-\frac{3 m}{\beta}>0,
$$

where the last inequality follows from the fact that $m=\lfloor\beta\rfloor$.

Therefore, the form is increasing in the variable $a_{0}$ and so the minimum is reached at the smallest possible value of $a_{0}$, i.e., at $a_{0}=1$. From now on we will consider $a_{0}=1$.

2) Difference $\Delta_{a_{1}}$. The inspected difference is

$$
\Delta_{a_{1}}=\frac{2 a_{1}+1}{\beta}+c_{1}+c_{1} \frac{a_{2}}{\beta}+c_{2} \frac{a_{3}}{\beta}+c_{3} \frac{a_{4}}{\beta} .
$$

Since $c_{1}<0, c_{2}<0, c_{3}>0$ we have

$$
\Delta_{a_{1}} \geq \frac{2 a_{1}}{\beta}-\frac{1}{\beta^{2}}-\frac{m}{\beta}\left(\frac{1}{\beta}+\frac{1}{\beta^{2}}\right)-\frac{m}{\beta}\left(\frac{2}{\beta}-\frac{1}{\beta^{2}}-\frac{2}{\beta^{3}}-\frac{1}{\beta^{4}}\right) \frac{2 a_{1}}{\beta}-\frac{3 m+1}{\beta^{2}} .
$$

The right side of the inequality is strictly greater than zero for $a_{1} \geq 2$. The minimum is therefore reached for either $a_{1}=0, a_{1}=1$ or $a_{1}=2$.

3) Difference $\Delta_{a_{2}}$. The inspected difference is

$$
\Delta_{a_{2}}=\frac{2 a_{2}+1}{\beta^{2}}+c_{2}+c_{1} \frac{a_{1}}{\beta}+c_{1} \frac{a_{3}}{\beta^{2}}+c_{2} \frac{a_{4}}{\beta^{2}} .
$$

For the difference $\Delta_{a_{2}}:=Q_{2}\left(a_{4}, a_{3}, a_{2}+1, a_{1}, a_{0}\right)-Q_{2}\left(a_{4}, a_{3}, a_{2}, a_{1}, a_{0}\right)$ one needs to consider only $a_{2} \leq m-1$. Since $c_{1}, c_{2}<0$, we obtain

$$
\Delta_{a_{2}} \leq \frac{2 m-1}{\beta^{2}}+c_{2}=\frac{2 m-1}{\beta^{2}}-\frac{2 m-1}{\beta^{2}}-\frac{1}{\beta^{4}}=-\frac{1}{\beta^{4}}<0 .
$$

The quadratic form is decreasing in the variable $a_{2}$ and the minimum is reached for the highest possible (w.r.t. Parry's condition) value of $a_{2}$.

4) Difference $\Delta_{a_{3}}$. The inspected difference is

$$
\Delta_{a_{3}}=\frac{2 a_{3}+1}{\beta^{3}}+c_{3}+c_{2} \frac{a_{1}}{\beta}+c_{1} \frac{a_{2}}{\beta^{2}}+c_{1} \frac{a_{4}}{\beta^{3}} .
$$

We have shown that the minimum of the quadratic form is reached for $a_{1} \in\{0,1,2\}$. It is suitable to discuss the cases $a_{1} \in\{0,1\}$ and $a_{1}=2$ separately. 
Let us assume $a_{1} \in\{0,1\}$. Since $a_{2}, a_{4} \leq m<\beta$ and $c_{1}, c_{2}<0$ we have

$$
\Delta_{a_{3}} \geq \frac{1}{\beta^{3}}+c_{3}+\frac{c_{2}}{\beta}+\frac{c_{1}}{\beta}+\frac{c_{1}}{\beta^{2}}=\frac{2(m-1)}{\beta^{4}}+\frac{1}{\beta^{6}}>0 .
$$

This means that for $a_{1} \in\{0,1\}$ the minimum of the form is reached for $a_{3}=0$. Moreover, we already know that the minimum is reached for the highest possible value of $a_{2}$ (w.r.t. Parry's condition), therefore, we will inspect following candidates for the minimum $\left(a_{4}, 0, m-1,1,1\right)$ and $\left(a_{4}, 0, m, 0,1\right)$.

The discussion for $a_{1}=2$ will be postponed for this moment.

5) Difference $\Delta_{a_{4}}$. The inspected difference is

$$
\Delta_{a_{4}}=\frac{2 a_{4}+1}{\beta^{4}}+c_{4}+c_{3} \frac{a_{1}}{\beta}+c_{2} \frac{a_{2}}{\beta^{2}}+c_{1} \frac{a_{3}}{\beta^{3}} .
$$

For the quintuple $\left(a_{4}, 0, m-1,1,1\right)$ we obtain

$$
\Delta_{a_{4}} \geq \frac{1}{\beta^{4}}+c_{4}+c_{3} \frac{1}{\beta}+c_{2} \frac{m-1}{\beta^{2}}>0 .
$$

Hence we have the first candidate for the point where the form $Q_{2}\left(a_{4}, a_{3}, a_{2}, a_{1}, a_{0}\right)$ reaches its minimum

$$
q_{1}:=(0,0, m-1,1,1) .
$$

For the quintuple $\left(a_{4}, 0, m, 0,1\right)$ we obtain

$$
\Delta_{a_{4}} \leq \frac{2 m+1}{\beta^{4}}+c_{4}+c_{2} \frac{m}{\beta^{2}}<0 .
$$

Second candidate for the point where the form $Q_{2}\left(a_{4}, a_{3}, a_{2}, a_{1}, a_{0}\right)$ reaches its minimum is

$$
q_{2}:=(m, 0, m, 0,1) .
$$

Here, we get back to the case $a_{1}=2$. We inspect the difference $\Delta_{a_{4}}$ for the quintuple $\left(a_{4}, a_{3}, a_{2}, 2,1\right)$. Note that due to the Parry's condition $a_{2} \leq m-1$. We can estimate

$$
\Delta_{a_{4}} \geq \frac{1}{\beta^{4}}+c_{4}+c_{3} \frac{2}{\beta}+c_{2} \frac{m-1}{\beta^{2}}+c_{1} \frac{m}{\beta^{3}}=\frac{2}{\beta^{3}}+\frac{2 m-1}{\beta^{4}}-\frac{3}{\beta^{5}}>0 .
$$

Hence we have to inspect the candidate $\left(0, a_{3}, a_{2}, 2,1\right)$.

We treat separately the case $a_{3}=m$, which implies $a_{2}=0$. So we have the third candidate

$$
q_{3}:=(0, m, 0,2,1)
$$

and the cases $a_{3} \leq m-1$, where according to the negativity of $\Delta_{a_{2}}$ the value of corresponding variable is $a_{2}=m-1$. The difference $\Delta_{a_{3}}$ for the quintuple $\left(0, a_{3}, m-1,2,1\right)$ is

$$
\Delta_{a_{3}}=\frac{2 a_{3}+1}{\beta^{3}}+c_{3}+c_{2} \frac{2}{\beta}+c_{1} \frac{m-1}{\beta^{2}}=\frac{2 a_{3}-2 m+6}{\beta^{3}}+\frac{1-m}{\beta^{4}}-\frac{2}{\beta^{5}}-\frac{1}{\beta^{6}} .
$$


It is easy to see that for $a_{3} \leq m-3$ the $\Delta_{a_{3}}<0$ and that for $a_{3} \geq m-2$ the $\Delta_{a_{3}}>0$. Therefore the fourth candidate for the minimum is

$$
q_{4}:=(0, m-2, m-1,2,1) .
$$

By computing the values of the quadratic form $Q_{2}$ in the points $q_{1}, q_{2}, q_{3}$ and $q_{4}$ we obtain

$$
\frac{(\beta+1)^{2}}{\beta 4}=Q_{2}\left(q_{1}\right)<\min \left\{Q_{2}\left(q_{2}\right), Q_{2}\left(q_{3}\right), Q_{2}\left(q_{4}\right)\right\} .
$$

Therefore, $\operatorname{Min}_{5}^{2}=\beta^{-4}(\beta+1)^{2}$ for $m \geq 3$.

Determining $\mathrm{Max}_{3}$. Similarly as in the previous part of the proof we will now inspect the first differences of the quadratic form $Q_{1}$ in the individual variables.

From the definition of $\operatorname{Max}_{3}$ we need to determine the maximal value of $Q_{1}$ over the set $\mathcal{S}_{1}$, which allows the coefficients $a_{0}, a_{1}, a_{2}$ take any values in $\{0,1, \ldots, m\}$ with the admissibility condition. However, it is sufficient to consider $a_{0}>0$, since $Q_{1}$ reaches its maximum on such a point. Otherwise, we would have

$$
\operatorname{Max}_{3}=\left|0+a_{1} \beta^{\prime}+a_{2}\left(\beta^{\prime}\right)^{2}\right|=\left|\beta^{\prime}\right|\left|a_{1}+a_{2} \beta^{\prime}\right| \leq\left|\beta^{\prime}\right| \operatorname{Max}_{2}<\operatorname{Max}_{2},
$$

which is in contradiction with $\operatorname{Max}_{2} \leq \operatorname{Max}_{3}$.

The inspection of the difference $\Delta_{a_{0}}$ of the quadratic form $Q_{1}$ is very similar to the one of $Q_{2}$ in the first part of the proof. We have

$$
\Delta_{a_{0}}=2 a_{0}+1+a_{1} c_{1}+a_{2} c_{2},
$$

$a_{0} \geq 1, c_{1}<0$ and $c_{2}<0$. Hence

$$
\Delta_{a_{0}} \geq 3+m c_{1}+m c_{2} \geq 3-\frac{3 m}{\beta}>0,
$$

the form is increasing in the variable $a_{0}$ and so the maximum is reached for the highest possible value of $a_{0}$ (w.r.t. Parry's condition).

Since

$$
\Delta_{a_{1}}=\frac{2 a_{1}+1}{\beta}+c_{1} a_{0}+c_{1} \frac{a_{2}}{\beta}
$$

is a linear function in the variable $a_{1}$ and the coefficient $\frac{2}{\beta}$ at $a_{1}$ is positive, the difference $\Delta_{a_{1}}$ is either positive for all values of $a_{1}$ or negative for all values of $a_{1}$ or negative for some initial values of $a_{1}$ and then positive, regardless of the values of $a_{0}$ and $a_{2}$.

Anyway, the maximum in the variable $a_{1}$ is reached for some extremal value of $a_{1}$. The same reasoning works also for the maximum in the variable $a_{2}$. 
One can easily see that there are only six candidates fulfilling preceding conditions on the values of the variables $a_{2}, a_{1}$ and $a_{0}$ :

\begin{tabular}{c|ccc} 
& $a_{2}$ & $a_{1}$ & $a_{0}$ \\
\hline$q_{1}$ & 0 & 0 & $m$ \\
$q_{2}$ & 0 & $m-1$ & $m$ \\
$q_{3}$ & 0 & $m$ & 1 \\
$q_{4}$ & $m-1$ & $m-1$ & $m$ \\
$q_{5}$ & $m-1$ & $m$ & 1 \\
$q_{6}$ & $m$ & 0 & $m$
\end{tabular}

Finally, by computing the values of the quadratic form $Q_{1}\left(a_{2}, a_{1}, a_{0}\right)$ in the points $q_{1}, \ldots, q_{6}$ we have

1. $Q_{1}(0,0, m)=m^{2}$.

2. $Q_{1}(0, m-1, m)=m^{2}+\frac{(m-1)^{2}}{\beta}-m(m-1)\left(\frac{1}{\beta}+\frac{1}{\beta 2}\right)<m^{2}$.

3. $Q_{1}(0, m, 1)=1+\frac{m^{2}}{\beta}-m\left(\frac{1}{\beta}+\frac{1}{\beta^{2}}\right)<m^{2}$.

4. $Q_{1}(m-1, m-1, m)=m^{2}+\frac{(m-1)^{2}}{\beta}+\frac{(m-1)^{2}}{\beta^{2}}-m(m-1)\left(\frac{1}{\beta}+\frac{1}{\beta^{2}}\right)-$

$$
-(m-1)^{2}\left(\frac{1}{\beta^{2}}+\frac{1}{\beta^{3}}\right)-m(m-1)\left(\frac{2}{\beta}-\frac{1}{\beta^{2}}-\frac{2}{\beta^{3}}-\frac{1}{\beta^{4}}\right)<m^{2} .
$$

5. $Q_{1}(m-1, m, 1)=1+\frac{m^{2}}{\beta}+\frac{(m-1)^{2}}{\beta^{2}}-m\left(\frac{1}{\beta}+\frac{1}{\beta^{2}}\right)-m(m-1)\left(\frac{1}{\beta^{2}}+\frac{1}{\beta^{3}}\right)-$

$$
-(m-1)\left(\frac{2}{\beta}-\frac{1}{\beta^{2}}-\frac{2}{\beta^{3}}-\frac{1}{\beta^{4}}\right)<m^{2} .
$$

6. $Q_{1}(m, 0, m)=m^{2}+\frac{m^{2}}{\beta^{2}}-m^{2}\left(\frac{2}{\beta}-\frac{1}{\beta^{2}}-\frac{2}{\beta^{3}}-\frac{1}{\beta^{4}}\right)<m^{2}$.

Therefore, $\operatorname{Max}_{3}^{2}=m^{2}$ and the proposition is proved.

\section{Combinatorial properties of $\beta$-integers}

It is known [Thu89] that the set $\mathbb{Z}_{\beta}$ has no accumulation points and that the distances between adjacent points in $\mathbb{Z}_{\beta}$ take values in the set $\left\{\sum_{i=1}^{\infty} \frac{t_{i+k}}{\beta^{i}} \mid k \in \mathbb{N}_{0}\right\}$ provided that $\beta$ is a Parry number. For $\beta$ with the Rényi expansion $d_{\beta}(1)=m 11$ the distances between adjacent points of $\mathbb{Z}_{\beta}$ take three values, namely $1, \frac{1}{\beta}+\frac{1}{\beta^{2}}, \frac{1}{\beta}$. If we code these distances by letters $a, b, c$, respectively, the tiling of the positive real line by the non-negative elements of $\mathbb{Z}_{\beta}$ can be represented as an infinite word $u_{\beta}:=u_{0} u_{1} u_{2} \cdots$ in a ternary alphabet $\{a, b, c\}$. An example of the tiling of the real line by $\mathbb{Z}_{\beta}$ for $m=2$, and the corresponding infinite word $u_{\beta}$ is shown on Figure 1. 


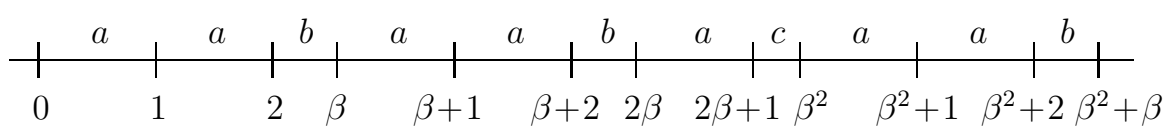

Fig. 1: The set $\mathbb{Z}_{\beta} \cap[0,+\infty)$ for $d_{\beta}(1)=211$ and the corresponding word $u_{\beta}$.

The infinite word $u_{\beta}$ for $d_{\beta}(1)=m 11$ is invariant under the primitive substitution [Fab95]

$$
\begin{aligned}
a & \mapsto a^{m} b, \\
b & \mapsto a c, \quad \text { where } a^{m} \text { stands for } \underbrace{a a \cdots a}_{m \text { times }} . \\
c & \mapsto a,
\end{aligned}
$$

For example, if $m=2$, then we generate the fixed point of the substitution by

$$
a \mapsto a a b \mapsto a a b a a b a c \mapsto a a b a a b a c a a b a a b a c a a b a \mapsto \cdots
$$

and we obtain the infinite word $u_{\beta}$ from Figure 1.

For the description of combinatorial properties of an infinite word, crucial is the notion of a factor. We say that a finite word $w=w_{0} w_{1} \ldots w_{n-1}$ is a factor of length $n$ of the infinite word $u=u_{0} u_{1} u_{2} \cdots$, if for some $i$ we have $w_{0} w_{1} \cdots w_{n-1}=u_{i} u_{i+1} \cdots u_{i+n-1}$. The function that to an integer $n$ associates the number $\mathcal{C}(n)$ of different factors of length $n$ in the infinite word $u$ is called the complexity of $u$. It is known that the complexity of the infinite word $u_{\beta}$ is $\mathcal{C}(n)=2 n+1$ for all $\beta$ such that $d_{\beta}(1)=m 11$. A proof can be found in [FMP04].

For $m=1$, the infinite word $u_{\beta}$ is called the Tribonacci word. It is known [AR91] that it is an Arnoux-Rauzy word, which means that for every length $n \in \mathbb{N}$ there exists a unique factor $w$ of $u_{\beta}$ of length $n$, such that $a w, b w$ and $c w$ are factors of $u_{\beta}$, and there exists a unique factor $v$ of $u_{\beta}$ of length $n$, such that $v a, v b$ and $v c$ are factors of $u_{\beta}$.

It is known that if an infinite word is Arnoux-Rauzy, then the set of its factors is invariant under mirror image, i.e., with every factor $w_{0} w_{1} \cdots w_{n-1}$ the infinite word contains the factor $w_{n-1} \cdots w_{1} w_{0}$. As a consequence, the infinite word $u_{\beta}$ for $m \geq 2$ cannot be Arnoux-Rauzy, because its set of factors is not stable under mirror image. This can be seen easily from the substitution (77) under which $u_{\beta}$ is invariant: $c a^{m} b$ is a factor of $u_{\beta}$, while $b a^{m} c$ is not.

Although the word $u_{\beta}$ is for $m \geq 2$ not Arnoux-Rauzy, it is still true that for every length $n \in \mathbb{N}$ there exists a unique factor $w$ of $u_{\beta}$ of length $n$, such that $a w, b w$ and $c w$ are factors of $u_{\beta}$. However, one does not have a word $v$ which could be extended with all the three letters to the right.

The invariance of the set of factors of the infinite word $u_{\beta}$ decides about a property of the image of $\mathbb{Z}_{\beta}$ under the field isomorphism ${ }^{\prime}: \mathbb{Q}(\beta) \mapsto \mathbb{Q}\left(\beta^{\prime}\right)$, the so-called Rauzy fractal. Figure 2 shows the set

$$
\mathbb{Z}_{\beta}^{\prime}=\left\{\sum_{i=0}^{k} a_{i} \beta^{i} \mid \sum_{i=0}^{k} a_{i} \beta^{i} \in \mathbb{Z}_{\beta}\right\}
$$

drawn in the complex plane, for the cases $m=1, m=2$. Bernat in [Ber05] has shown that if $m=1$, the closure of $\mathbb{Z}_{\beta}^{\prime}$ is a set centrally-symmetric with respect to the point $c=\frac{1}{2}\left(1-\beta^{\prime}\right)^{-1}$. As it can be seen from Figure $2, \mathbb{Z}_{\beta}^{\prime}$ for $m=2$ does not have this property. 


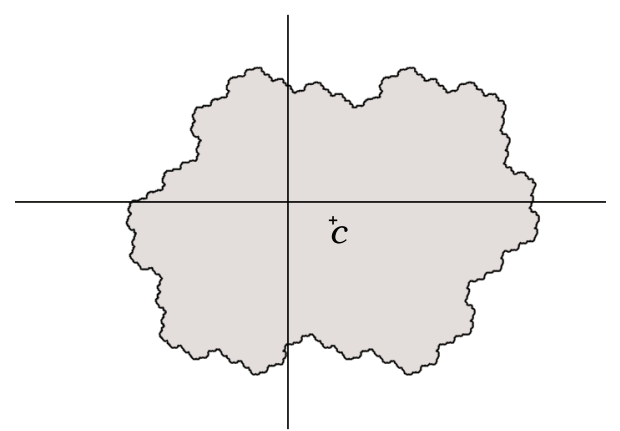

(a) Tribonacci numeration system.

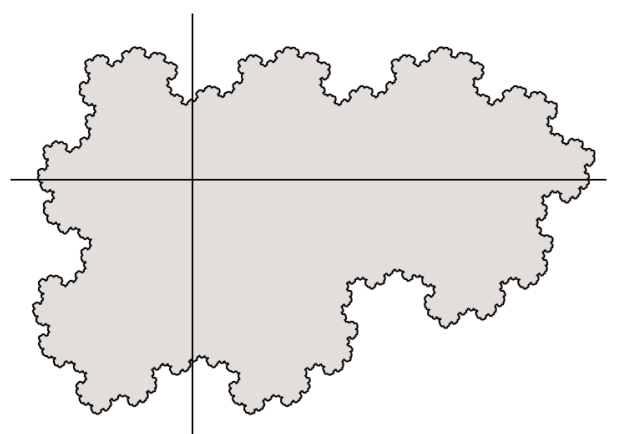

(b) Numeration system with minimal polynomial $x^{3}-2 x^{2}-x-1$.

Fig. 2: Central tiles in conjugated plane.

\section{Acknowledgements}

The authors acknowledge financial support of Czech Science Foundation GA ČR 201/05/0169, and by the grant LC06002 of the Ministry of Education, Youth, and Sports of the Czech Republic.

\section{References}

[ABF04] Shigeki Akiyama, Frédérique Bassino, and Christiane Frougny. Automata for arithmetic Meyer sets. In LATIN 2004: Theoretical informatics, volume 2976 of Lecture Notes in Comput. Sci., pages 252-261. Springer, Berlin, 2004.

[AFMP03] P. Ambrož, C. Frougny, Z. Masáková, and E. Pelantová. Arithmetics on number systems with irrational bases. Bull. Belg. Math. Soc. Simon Stevin, 10(suppl.):641$659,2003$.

[Aki99] Shigeki Akiyama. Self affine tiling and Pisot numeration system. In K. Györy and S. Kanemitsu, editors, Number theory and its applications (Kyoto, 1997), volume 2 of Dev. Math., pages 7-17. Kluwer Acad. Publ., Dordrecht, 1999.

[Aki00] Shigeki Akiyama. Cubic Pisot units with finite beta expansions. In Algebraic number theory and Diophantine analysis (Graz, 1998), pages 11-26. de Gruyter, Berlin, 2000.

[AR91] Pierre Arnoux and Gérard Rauzy. Représentation géométrique de suites de complexité $2 n+1$. Bull. Soc. Math. France, 119(2):199-215, 1991.

[ARS04] Shigeki Akiyama, Hui Rao, and Wolfgang Steiner. A certain finiteness property of Pisot number systems. J. Number Theory, 107(1):135-160, 2004. 
[AS98] Shigeki Akiyama and Taizo Sadahiro. A self-similar tiling generated by the minimal Pisot number. In Proceedings of the 13th Czech and Slovak International Conference on Number Theory (Ostravice, 1997), volume 6, pages 9-26, 1998.

[Ber05] Julien Bernat. Propriétés arithmétiques de la $\beta$-numération. PhD thesis, Univesité de la Méditeranée, 2005.

[BFGK98] Čstmír Burdík, Christiane Frougny, Jean-Pierre Gazeau, and Rudolf Krejcar. Betaintegers as natural counting systems for quasicrystals. J. Phys. A, 31(30):6449-6472, 1998.

[Fab95] Stéphane Fabre. Substitutions et $\beta$-systèmes de numération. Theoret. Comput. Sci., 137(2):219-236, 1995.

[FMP04] Christiane Frougny, Zuzana Masáková, and Edita Pelantová. Complexity of infinite words associated with beta-expansions. Theor. Inform. Appl., 38(2):163-185, 2004. Corrigendum. Theor. Inform. Appl. 38 (2004), 269-271.

[FS92] Christiane Frougny and Boris Solomyak. Finite beta-expansions. Ergodic Theory Dynam. Systems, 12(4):713-723, 1992.

[Gji01] Nertila Gjini. A self-similar tiling generated by the Pisot number which is the root of the equation $x^{3}-x^{2}-1=0$. Osaka J. Math., 38(2):303-319, 2001.

[GMP04] L. S. Guimond, Z. Masáková, and E. Pelantová. Arithmetics of beta-expansions. Acta Arith., 112(1):23-40, 2004.

[Hol96] M. Hollander. Linear numeration systems, finite beta-expansions, and discrete spectrum of substitution dynamical systems. PhD thesis, Washington University, 1996.

[Mes00] Ali Messaoudi. Généralisation de la multiplication de Fibonacci. Math. Slovaca, $50(2): 135-148,2000$.

[Mes02] Ali Messaoudi. Tribonacci multiplication. Appl. Math. Lett., 15(8):981-985, 2002.

[Par60] W. Parry. On the $\beta$-expansions of real numbers. Acta Math. Acad. Sci. Hungar., 11:401-416, 1960.

[Thu89] William P. Thurston. Groups, tilings, and finite state automata. AMS Colloquium Lecture Notes, 1989. 